\title{
Production of Good Manufacturing Practice Grade Equine Adipose- derived Mesenchymal Stem Cells for Therapeutic Use
}

Shashank Gowda, Aarya Hari, Basavaraj Chougule, Manoj Kumar Reddy, Abhishek Chandanan, Minita Sodhi, Nicole Koshy, Lyle Fonseca, Satish Totey*

Department of Stem Cell Research, Kasiak Research Private Limited, Thane-400610, Maharashtra, India

\begin{abstract}
Mesenchymal stem cells (MSC) are currently being evaluated in equine clinical studies for their potential to treat various disorders and injuries. Studies have shown that both autologous and allogeneic stem cells appear to be safe. However, an efficient cell expansion method that is reliable and cost-effective is warranted to provide off-the-shelf clinical grade stem cells. Our study aimed to determine optimum culture conditions for efficient large-scale stem cell expansion. We produced cGMP standard equine adipose derived mesenchymal stem cells on a large scale. Five different medium combinations-Dulbecco's modified Eagle's medium-knockout (DMEM-KO), alpha modified

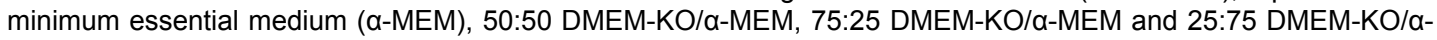
MEM - at seeding densities of $1000,2000,3000,4000$ and 5000 cells $/ \mathrm{cm}^{2}$, were used to determine the optimum culture conditions for large-scale production. Growth kinetics, immunophenotypes, karyotypes, morphology, trilineage differentiation, T-cell proliferation, virus positivity, pre-clinical toxicity and expression of pluripotency markers were analysed. Among the medium combinations and seeding densities tested, 25:75 DMEM-KO/a-MEM at a seeding density of $5000 \mathrm{cells} / \mathrm{cm}^{2}$ was found to be optimum for large-scale expansion. This medium combination gave a significantly higher cell yield than the other medium combinations, while preserving their stem cell characteristics and differentiation potential. The results indicated that the adoption of an appropriate culture system significantly improved cell yield, thus enabling the production of sufficient cells for therapeutic application in a cost-effective manner. The results also showed that the method of large-scale expansion requires minimal manipulation of cells, and it could be expanded ex vivo within two passages while retaining the characteristics of true stem cells.
\end{abstract}

Keywords: Adipose tissue; Mesenchymal stem cells; Ex vivo expansion; Clinical grade; Growth kinetics; Culture media; cGMP

Abbreviations: $a-M E M$ : Alpha Modified Minimum Essential Medium; 7-AAD: 7-Amino-Actinomycin D; AD-MSC: Adipose Derived Mesenchymal Stem Cells; cGMP: Current Good Manufacturing Practice; DPBS: Dulbecco's Phosphate-buffered Saline; DMEM-KO: Dulbecco's Minimum Essential Medium-knock Out DMSO: Dimethyl Sulfoxide; EDTA: Ethylenediaminetetraacetic Acid; FBS: Fetal Bovine Serum; MCB: Master Cell Bank; MSC: Mesenchymal Stem Cells; RT: Room Temperature; PBMCs: Peripheral Blood Mononuclear Cells; PHA: Phytohemagglutinin; WCB: Working Cell Bank

\section{Introduction}

Mesenchymal stem cells (MSCs) with their ability of self-renewal, ability to exert paracrine effect, its profound immunomodulatory properties and multi-lineage differentiation potential could be a very promising tool for regenerative medicine in equines [1-3]. There are several sources of MSCs available for therapeutic purpose in equines including bone marrow [4], amnion [5], adipose tissue [6,7] and umbilical cord [8]. These MSCs are tested for wide spectrum of clinical conditions such as tendon injury, ligament injury, osteoarthritis and exercise induced pulmonary haemorrhage [9-11]. MSCs represent a multipotent adult stem cells population and tend to home to sites of tissue growth to repair damaged tissue and enhance tissue regeneration. The expression of a variety of chemokine receptors or adhesion molecules on MSCs may account for their potential multiorgan homing capacity [12-14].

The use of MSC as cellular product for therapeutics requires standardized protocol for isolation and reliable quality control procedure. Most of the stem cell harvesting protocols that are currently being used for regenerative medicine in equine is not in accordance with the criteria of stringent cGMP guidelines and lack quality control parameters $[6,9,11]$. Stem cells that were reportedly tested for equine are mostly adipose derived stromal vascular fractions; bone marrow derived mononuclear cells or autologous mesenchymal stem cells. Although, it is not clear if autologous or allogeneic MSCs are preferable, but there are several indisputable advantages of allogeneic cells for use as off-the-shelf products thus avoiding the need for repeated bone marrow aspiration or collection of adipose tissue from each patient. This will avoid tissue culture delay before treatment [15]. Moreover, quality tests including sterility, animal safety, differentiation potential and immunosuppression viability, endotoxin and mycoplasma, is possible before the cells are released for therapeutic purpose.

However, irrespective of autologous or allogeneic MSCs, there is a growing perception that even under highly standardized culture conditions, long-term cultures evoke continuous changes in MSC, proliferation rate decreases that leads to replicative senescence, differentiation potential is affected, chromosomal instabilities may arise and molecular changes may acquire that may affect therapeutic outcome [16].

In view of the potential application of stem cells in equine therapy,

*Corresponding author: Satish M Totey, Kasiak Research Private Limited, DIL Complex, Ghodbunder Road, Thane (W) 400610, Maharashtra, India, Tel: +91-2241173473; E-mail: satish.totey@kasiakresearch.com

Received September 24, 2013; Accepted October 31, 2013; Published November 02, 2013

Citation: Gowda S, Hari A, Chougule B, Reddy MK, Chandanan A, et al. (2013) Production of Good Manufacturing Practice Grade Equine Adipose-derived Mesenchymal Stem Cells for Therapeutic Use. J Stem Cell Res Ther 3: 154. doi:10.4172/2157-7633.1000154

Copyright: ( 2013 Gowda S, et al. This is an open-access article distributed unde the terms of the Creative Commons Attribution License, which permits unrestricted use, distribution, and reproduction in any medium, provided the original author and source are credited. 
there is growing interest in optimizing the expansion protocols to produce large quantities of stem cells in a cost-effective manner and without evoking replicative senescence. To date, there is inconsistency among laboratories concerning the type of media and supplementary factors for the successful isolation and expansion of AD-MSCs to obtain homogeneous cell populations in both in vivo experiments and clinical trials.

In this study, we have addressed the inconsistency by setting the optimum culture conditions for effective clinical grade production of large numbers of AD-MSCs in a cost- and time-effective manner with less manipulation and passages in order to avoid changes in MSCs. The results of this study are highly reproducible and consistent. This expansion method can be used to provide readily available and affordable equine cellular therapy.

\section{Materials and Methods \\ Isolation of adipose tissue}

Mesenchymal stem cells were isolated from the gluteal subcutaneous adipose tissue of eight healthy thoroughbred horses following the protocols approved by the Institutional Animal Ethics Committee (IAEC). Adipose tissue was collected as per the method described previously [7].

\section{Processing of adipose tissue}

Approximately 15-20 g of adipose tissue was obtained from three thoroughbred horses and placed in a $50-\mathrm{ml}$ polypropylene centrifuge tube containing sterile Dulbecco's phosphate-buffered saline (DPBS) (Invitrogen, Grand Island NY) supplemented with penicillin and streptomycin solution (Invitrogen) and transported to the central processing laboratory at $20-22^{\circ} \mathrm{C}$. All sample processing was done inside a class 100 biosafety hood in a class B cGMP: current good manufacturing practices, facility. Adipose tissues were washed thoroughly with DPBS, minced with scissors and subjected to digestion with freshly prepared $0.1 \%$ type 1 collagenase (MP Biomedical, Santa Ana, CA) for 30 minutes. To the digested adipose tissue, an equal volume of MSC culture medium, consisting of Dulbecco's modified Eagle's medium-knockout (DMEM-KO) (Invitrogen) supplemented with $10 \%$ fetal bovine serum (FBS), (Hyclone, South Logan UT) $2 \mathrm{ng} / \mathrm{ml}$ basic fibroblast growth factor (bFGF) (Invitrogen) and 1\% GlutaMAX (Invitrogen) was added to neutralize the enzyme. The suspension was then centrifuged at $2500 \mathrm{rpm}$ for 10 minutes. The resulting cell pellet was then resuspended in respective culture medium. The resulting cell suspension obtained was the stromal vascular fraction (SVF). The SVF was filtered through a $100-\mu \mathrm{m}$ filter (Millipore India, Bangalore, India), then through a $40-\mu \mathrm{m}$ filter (Millipore). Cells were counted and analysed by flow cytometry (FACSCalibur) (BD Bioscience, Franklin Lake, NJ).

\section{Medium optimization}

To determine the optimum culture conditions for equine $\mathrm{AD}$ MSCs, we evaluated five different medium combinations: (i) $100 \%$ DMEM-KO, (Invitrogen) (ii) 100\% alpha modified minimum essential medium ( $\alpha-M E M)$ (Invitrogen), (iii) combination of 50\% DMEM and 50\% $\alpha$-MEM (50:50 DMEM-KO- $\alpha$-MEM), (iv) 75\% DMEM and $25 \%$ $\alpha$-MEM (75:25 DMEM-KO- $\alpha$-MEM) and (v) 25\% DMEM-KO and 75\% a-MEM (25:75 DMEM-KO-a-MEM) supplemented with 10\% FBS, $1 \%$ GlutaMAX and $2 \mathrm{ng} / \mathrm{ml}$ bFGF.

Nucleated SVF cells were initially seeded in a 1-chamber stack (Corning plastic ware supplied by Sigma-Aldrich Chemical Pvt. Ltd.,
Bangalore, India) in five different media composition and at 85,000 to $100,000 \mathrm{cells} / \mathrm{cm}^{2}$ seeding density. All the cultures were incubated at $37^{\circ} \mathrm{C}$ in $5 \%$ humidified $\mathrm{CO}_{2}$. Media were changed every third day, and cells were trypsinized when they reached $\sim 90 \%$ confluence. Equine AD-MSCs were harvested with $0.05 \%$ trypsin plus $0.2 \%$ EDTA, washed with DPBS and counted with a haemocytometer at passage- $0(\mathrm{P}-0)$. The cells were aliquoted, frozen at the concentration of 1 million cells/vial and stored in liquid nitrogen at passage- 0 (P-0).

Subsequently, P-0 AD-MSCs were thawed and seeded into a T-175 flask (Corning) with five individual medium types and five different seeding densities, i.e. 1000, 2000, 3000, 4000 and $5000 \mathrm{cells} / \mathrm{cm}^{2}$. All cultures were incubated at $37^{\circ} \mathrm{C}$ in $5 \%$ humidified $\mathrm{CO}_{2}$. Media were changed every third day, and cells were passaged when they reached $\sim 90 \%$ confluence. Equine AD-MSCs were harvested with $0.05 \%$ trypsin plus $0.2 \%$ EDTA, washed with DPBS and counted with a haemocytometer. This experiment was done with three different donors in three replicates, up to three passages.

\section{Growth kinetics}

Growth kinetics was analysed by calculating the total number of population doublings and population doubling time as previously described [17-19].

\section{Immunophenotyping}

Cells cultured in the different basal media were analysed for MSCrelated cell surface markers by flow cytometric analysis as previously described [17-19]. Cells were stained with the following antibodies: mouse IgG1 conjugated to FITC, mouse anti-human CD34, FITC mouse anti-human CD45, anti-HLA-DR (BD Bioscience, San Jose, CA. and FITC CD44 (Acris Antibodies, Inc., Herford, Germany.) mouse IgG1 conjugated to PE, mouse anti-human CD90, and PE mouse antihuman IgG1 CD105 (BD Bioscience).

Cell viability was measured by flow cytometry analysis using 7-AAD. All analyses were standardized against negative control cells incubated with isotype-specific IgG1-PE and IgG1-FITC (BD Bioscience) At least 10,000 events were acquired on a FACSCalibur flow cytometer, and the results were analysed using CellQuest Express Pro software (BD Bioscience).

\section{Trilineage differentiation}

The potential for trilineage differentiation, i.e., adipocyte, osteocyte and chondrocyte differentiation, of equine AD-MSCs cultured in five different culture conditions and seeding densities was assessed as previously described [17-19]. Images were captured using a Nikon Eclipse 90i microscope (Nikon, Tokyo, Japan).

\section{Karyotype analysis}

Karyotyping of the AD-MSCs cultured in five different culture conditions and seeding densities was performed as previously described [17-19]. At least 20 metaphase spreads were analysed from each MSC preparation. Images were acquired using a Nikon Eclipse 90i microscope (Nikon) and analyzed using IKaros software. Cells were analyzed with a band resolution of 434 (ISCNH 1997).

\section{Immunosuppression assay}

Peripheral blood mononuclear cells (PBMCs) were isolated from whole blood samples from three donors using Histopaque (SigmaAldrich Chemical Pvt Ltd, Bangalore, India.) Equine AD-MSCs were inactivated with $5 \mu \mathrm{g} / \mathrm{ml}$ actinomycin D (Sigma) and co-cultured with 
PBMCs in a 96-well plate at ratios of 1:100 and 1:500 (MSC:PBMC). Phytohemagglutinin (PHA) (Sigma) $(10 \mu \mathrm{g} / \mathrm{ml})$ was added to the test and positive control wells for PBMC stimulation. Cultures were incubated in $200 \mu \mathrm{L}$ of MSC culture medium for 72 hours, and BrdU labelling reagent was added in the last 24 hours of the assay. Estimation of cell proliferation was conducted using the BrdU colorimetric cell proliferation kit (Roche Applied Sciences, Indianapolis, IN) as per the manufacturer's instructions. MSC-induced suppression of T-cell proliferation was calculated in terms of percentage T-cell proliferation relative to the positive control, which was assumed to show $100 \%$ proliferation.

\section{Large scale expansion}

Preparation of master cell bank: Approximately 15-20 g of adipose tissue was again obtained from five thoroughbred horses and processed as per method given above. The SVF was seeded in a 1-chamber stack (Corning) in optimum media selected after media optimization experiment. Here we used 25:75 DMEM-KO: $\alpha$-MEM supplemented with $10 \% \mathrm{FBS}, 1 \%$ GlutaMAX and $2 \mathrm{ng} / \mathrm{ml}$ bFGF as an optimized MSC culture medium. The SVF was seeded at a density of $85,000-100,000$ cells $/ \mathrm{cm}^{2}$. All the cultures were incubated at $37^{\circ} \mathrm{C}$ in $5 \%$ humidified $\mathrm{CO}_{2}$. The medium was changed every third day, and cells were passaged at $\sim 90 \%$ confluence. AD-MSCs were harvested with $0.05 \%$ trypsin plus $0.2 \%$ (EDTA), washed with DPBS and counted with a haemocytometer. The cells from the individual donors were tested for quality parameters. The cells were aliquoted, frozen at the concentration of 1 million cells/vial and stored in liquid nitrogen as a master cell bank (MCB) at passage-0 (P-0).

Preparation of working cell bank: Four frozen vials of MCB from four individual donors were thawed and cells were pooled together. Mixed donor cells from MCB were then seeded in 5-chamber stack (Corning) with optimized MSC medium at the seeding density of 5000 cells $/ \mathrm{cm}^{2}$.

All the cultures were incubated at $37^{\circ} \mathrm{C}$ and $5 \%$ humidified $\mathrm{CO}_{2}$. The medium was changed every third day, and the cells were harvested at $\sim 90 \%$ confluence with $0.05 \%$ trypsin plus $0.2 \%$ EDTA, washed with DPBS and counted with a haemocytometer. Cells of the WCB were tested for quality parameters as described above and were frozen at the concentration of 10 million cells/vial and stored in liquid nitrogen at passage-1 (P-1).

Final product expansion: Frozen vials of WCB were thawed, and cells were seeded at a seeding density of $5000 \mathrm{cells} / \mathrm{cm}^{2}$ in a 10 -chamber stack (Corning) containing optimized MSC medium. All cultures were incubated at $37^{\circ} \mathrm{C}$ in $5 \%$ humidified $\mathrm{CO}_{2}$. The medium was changed every fourth day, and the cells were harvested at $\sim 90 \%$ confluence. The cells were harvested with $0.05 \%$ trypsin plus $0.2 \%$ EDTA, washed with injectable Plasmalyte-A ( $\mathrm{pH}$ 7.3-7.4) and counted with a haemocytometer. Final product was then frozen at a concentration of 10 million cells/vial and stored in liquid nitrogen at passage-2 (P-2).

Quality control testing: The MCB, WCB and final product were tested for quality parameters as follows. Morphology: small slender fibroblast like appearance; immunophenotypic analysis: CD34 $(<2 \%)$, CD44 (>85\%), CD45 (<2\%), CD90 (>85\%), CD105 (>85\%), HLA-DR $(<2 \%)$, 7-aminoactinomycin D (7-AAD) $(>80 \%)$; bacterial endotoxin: Lal Test (Charles River, Wilmington, MA(specification $<0.5 \mathrm{EU} / \mathrm{ml}$ ), mycoplasma (PCR, Venor Gem, Minerva Biolab, Berlin, Germany); pH (7.0-7.8); sterility, 7-day sterility (BactAlert 3D automated system, Biomeurix, France); trilineage differentiation: osteoblasts, adipocytes, chondrocytes; karyotyping ( $\mathrm{G}$ banding); and viral screening for equine influenza virus (EIV-A), equine herpes virus-1 (EHV-1), EHV-2, EHV3, EHV-4, EHV-5, equine arteritis virus, equine infectious anaemia virus and West Nile virus by polymerase chain reaction (PCR). Batches were released for clinical application if they satisfied internally set quality parameters.

Freezing and thawing of final product: AD-MSCs from final product at passage- 2 were washed with Plasmalyte-A at $\mathrm{pH}$ 7.37.4. Ten million cells were suspended in cryoprotectant solution that consists of $2 \mathrm{ml}$ of Plasmalyte-A (Baxter India), USP grade 10\% dimethyl sulphoxide (DMSO) (Gaylord, Tuscaloosa, AL), 5\% qualified equine serum (Hyclone). Cells were frozen in programmable freezer and stored in vapour phase of liquid nitrogen.

Final product was thawed by placing vial in a $37^{\circ} \mathrm{C}$ water bath for 1 min or until cells begin to thaw. Cells were further diluted by adding $3 \mathrm{ml}$ of Plasmalyte-A in order to bring total volume to $5 \mathrm{ml}$. After diluting the cells each $5 \mathrm{ml}$ final product consists of 10 million cells, Plasmalyte-A, 3.3\% DMSO and 1.6\% qualified equine serum.

\section{Pre-clinical safety study}

Fourteen days acute toxicity in Wistar rats and Swiss Albino mice by intravenous route at the dose of 20 million cells $/ \mathrm{kg}$ body weight was carried out.

\section{Statistical analysis}

Statistical analysis was carried out using the GraphPad Prism (Version 5.03) software (GraphPad Software, Inc., La Jolla, CA). Data were presented as means \pm SEM For all experiments; a oneway ANOVA followed by either a Dunnett's or Bonferroni's post hoc test was performed. $\mathrm{P}$ values of $<0.05$ were considered statistically significant.

\section{Results}

\section{Isolation of stromal vascular fraction}

Adipose tissue samples from all the eight different healthy donors were obtained and processed for isolation of SVF. The number of nucleated cells in SVF from the eight different donors were consistent and without significant variation. Averages of $3.6 \times 10^{6} \pm 0.13$ nucleated cells per gram of fat tissue were obtained (Table 1 ).

\section{Selection of optimum basal medium for expansion and growth characteristics of equine AD-MSCs}

To determine the optimum basal medium for long-term culture and large-scale expansion of equine MSCs from equine adipose tissue, nucleated SVF cells were isolated and seeded in five different basal medium at 80,000 to 100,000 cells $/ \mathrm{cm}^{2}$ seeding density in 1 -chamber stacks. Details of the cell harvested at passage-0 (P-0) from 1-chamber stacks are given in Table 2. Harvested AD-MSCs were used for subsequent media optimization studies.

A total of three passages were analysed in three replicates using three different donor MSCs. Selection of two main basal medias such as DMEM-KO and $\alpha$-MEM was selected on the basis of our earlier reports [17-19] where we found that DMEM-KO and a-MEM medium were optimal culture medias compared to DMEM-low glucose (DMEMLG), DMEM-high glucose (DMEM-HG) and DMEM-F12 for longterm culture of human MSC derived from adipose tissue, bone marrow and dental pulp [17-19]. Current experiment was designed to further analyse synergistic effect of both the medias on equine MSCs. 
Citation: Gowda S, Hari A, Chougule B, Reddy MK, Chandanan A, et al. (2013) Production of Good Manufacturing Practice Grade Equine Adiposederived Mesenchymal Stem Cells for Therapeutic Use. J Stem Cell Res Ther 3: 154. doi:10.4172/2157-7633.1000154

Page 4 of 11

The equine AD-MSCs cultured in all different medias maintained a normal phenotype, showing a typical small spindle-shaped morphology in all the passages and replicates with consistent results (Figure 1A). There was no significant difference in morphological characteristics among the AD-MSCs cultured in five different culture conditions and seeding densities.

Proliferation rate: To assess the proliferation rate of equine ADMSC under different culture conditions, growth curves were plotted. For this, the total number of cells at each passage was calculated as a ratio of total number of cells harvested to total number of cells seeded. The growth curve varied considerably between the different adipose tissue samples obtained from the donors and each of the media tested. We observed that proliferation rate was significantly influenced by culture condition and seeding density. In this experiment proliferation rate was significantly higher in $\alpha$-MEM or combination of medium containing higher percentage of $\alpha$-MEM $(\mathrm{P}<0.05)$. At the end of the passage 3, maximum cumulative cell yield was significantly higher at the seeding density of 5000 cells $/ \mathrm{cm}^{2}$ in 25:75 DMEM-KO: $a-M E M(4.4$

\begin{tabular}{|c|c|c|c|}
\hline Used for & Donor no. & Weight of fat (in gms) & No of cells nucleated cells/g of fat \\
\hline \multirow{3}{*}{ Media optimization study } & 1 & 15 & $3.3 \times 10^{6} / \mathrm{g}$ \\
\hline & 2 & 20 & $2.9 \times 10^{6} / g$ \\
\hline & 3 & 14 & $3.3 \times 10^{6} / \mathrm{g}$ \\
\hline \multirow{5}{*}{ Ex vivo expansion } & 4 & 17 & $3.7 \times 10^{6} / \mathrm{g}$ \\
\hline & 5 & 22 & $3.8 \times 10^{6} / \mathrm{g}$ \\
\hline & 6 & 20 & $3.8 \times 10^{6} / \mathrm{g}$ \\
\hline & 7 & 22 & $4.0 \times 10^{6} / \mathrm{g}$ \\
\hline & 8 & 20 & $4.0 \times 10^{6} / \mathrm{g}$ \\
\hline
\end{tabular}

Table 1: Yield of nucleated stromal vascular fraction from the equine adipose tissue isolated from the individual donors.

\begin{tabular}{|c|c|c|c|c|c|}
\hline Donor no. & DMEM-KO & $\alpha-M E M$ & 50:50 DMEM-KO: $\alpha-M E M$ & 75:25 DMEM-KO: $\alpha-M E M$ & 25:75 DMEM-KO: $\alpha-M E M$ \\
\hline 1 & $87 \times 10^{6}$ & $96 \times 10^{6}$ & $80 \times 10^{6}$ & $83 \times 10^{6}$ & $110 \times 10^{6}$ \\
\hline 2 & $90 \times 10^{6}$ & $110 \times 10^{6}$ & $87 \times 10^{6}$ & $89 \times 10^{6}$ & $115 \times 10^{6}$ \\
\hline 3 & $70 \times 10^{6}$ & $108 \times 10^{6}$ & $90 \times 10^{6}$ & $100 \times 10^{6}$ & $120 \times 10^{6}$ \\
\hline
\end{tabular}

Table 2: Total yield of AD-MSC at passage- 0 from individual donors cultured in 5 different media composition.
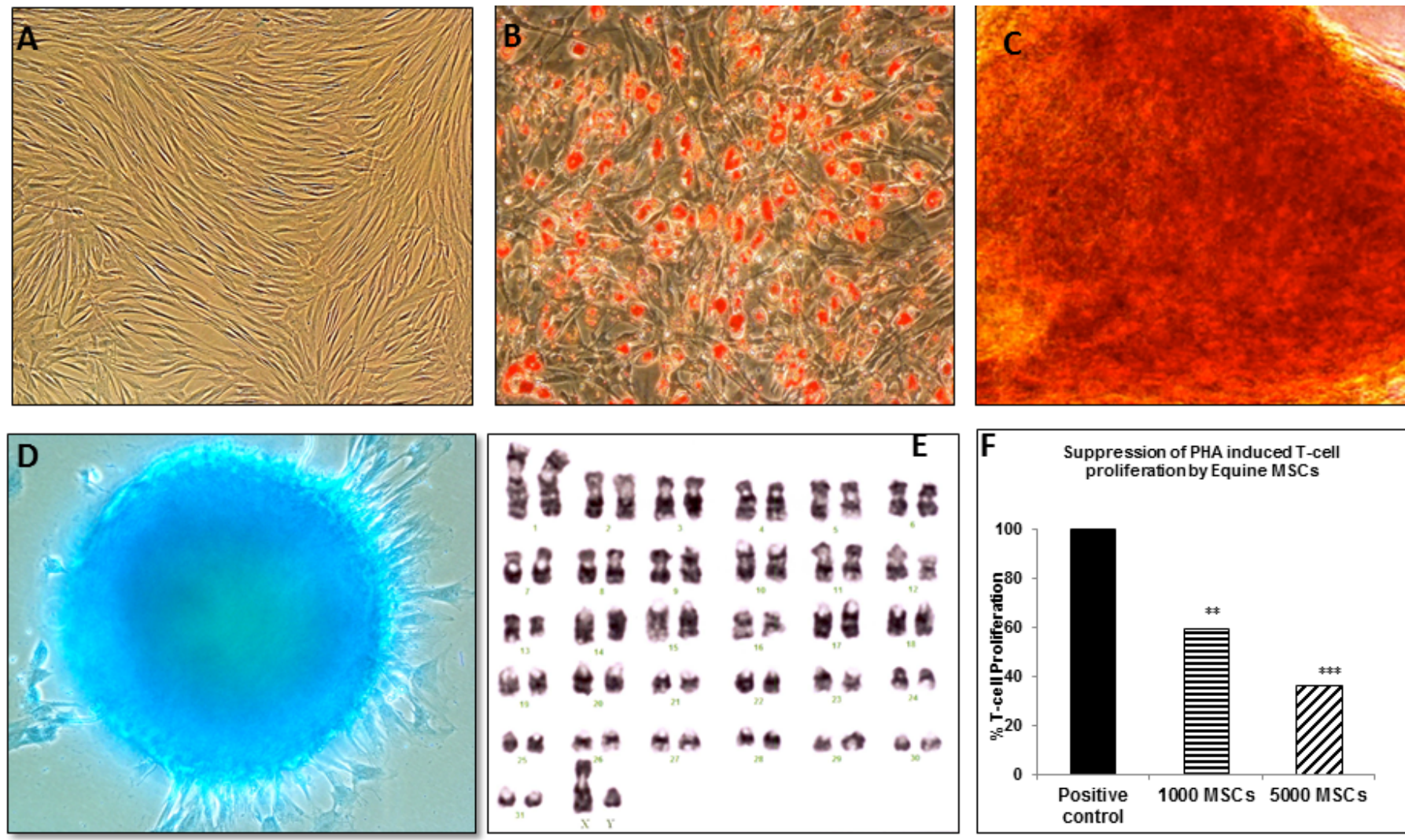

Figure 1: Representative pictures of: (A) phase-contrast microscopy of equine AD-MSC morphology depicting small fibroblast-like spindle-shaped cells (10x). (B) Adipogenesis was detected by neutral oil droplet formation stained with Oil Red O at day $24(10 \times)$. (C) Osteogenesis was confirmed by mineralized matrix deposition stained with Alizarin Red at day 21(10x). (D) Chondrogenesis was detected by the presence of proteoglycans with Alcian Blue staining (10x). (E) Cytogenetic stability of cells under three passages under various culture conditions. (F) Suppression of PHA induced T-cell proliferation by equine AD-MSC at dose dependent manner. Results are average of three replicates. ${ }^{* *}$ indicates $\mathrm{P}<0.01,{ }^{, * *}$ indicates $\mathrm{P}<0.001$ compared to the positive control. 
Citation: Gowda S, Hari A, Chougule B, Reddy MK, Chandanan A, et al. (2013) Production of Good Manufacturing Practice Grade Equine Adiposederived Mesenchymal Stem Cells for Therapeutic Use. J Stem Cell Res Ther 3: 154. doi:10.4172/2157-7633.1000154

Page 5 of 11

$\left.\times 10^{10}\right)$ and $\alpha$-MEM $\left(3.3 \times 10^{10}\right)$ compared to that of DMEM-KO alone $\left(1.4 \times 10^{10}\right)(\mathrm{P}<0.05)$. However, we did not observe synergistic effect of media combination on equine AD-MSC proliferation.

Seeding density: Effect of seeding density on expansion of equine $\mathrm{AD}-\mathrm{MSC}$ among the various media combination was tested. Equine AD-MSC were cultured in T175 flasks at 1000, 2000, 3000, 4000 and 5000 cells $/ \mathrm{cm}^{2}$ starting from passage- 1 and continued until passage- 3 . We observed seeding densities at 5000 cells $/ \mathrm{cm}^{2}$ provided the best starting condition for cell proliferation and overall yield $(\mathrm{P}<0.05)$. At passage-3, overall yield was significantly higher in $\alpha$-MEM alone (34.59 $\left.\times 10^{6}\right)$ or combination of 25:75 DMEM-KO: $\alpha$-MEM $\left(36.98 \times 10^{6}\right)$ at the seeding density of 5000 cells $/ \mathrm{cm}^{2}$ than DMEM-KO alone $(21.0 \times$ $\left.10^{6}\right)$, 50:50 DMEM-KO: $\alpha$-MEM $\left(30.85 \times 10^{6}\right)$ and 75:25 DMEM-KO: $\alpha-$ $\operatorname{MEM}\left(26.95 \times 10^{6}\right)$ in T175 culture flasks $(\mathrm{P}<0.05)$ (Figure 2$)$.

Cumulative population doubling: Most convenient parameter for documentation of long-term culture was number of passages. But that may lead to deceptive results and hence number of cumulative population doublings was calculated. In this experiment we did not observe significant difference in cumulative population doubling at the end of passage- 3 between the culture conditions. However, cumulative population doubling at seeding density of 3000, 4000 and 5000 cells/ $\mathrm{cm}^{2}$ was significantly less than cells seeded at $1000 \mathrm{cells} / \mathrm{cm}^{2}(\mathrm{P}<0.05)$. There was no significant difference in apoptosis rate analysed by betagalactosidase staining in all the culture conditions and seeding densities suggesting no significant replicative senescence during the passages. Cumulative population doublings at the seeding density of 5000 cells/ $\mathrm{cm}^{2}$ in all the media combination at the end of passage 3 ranged from 14.09 to 16.07 which is well within the accepted limit (Figure 3).

Population doubling time: The population doubling time of equine $\mathrm{AD}-\mathrm{MSC}$ in all the media combination was analysed. Results showed that media composition significantly influenced doubling time. Average population doubling time of the cell cultured in DMEM$\mathrm{KO}$ irrespective of seeding density at the end of passage- 3 was $37.31 \mathrm{~h}$
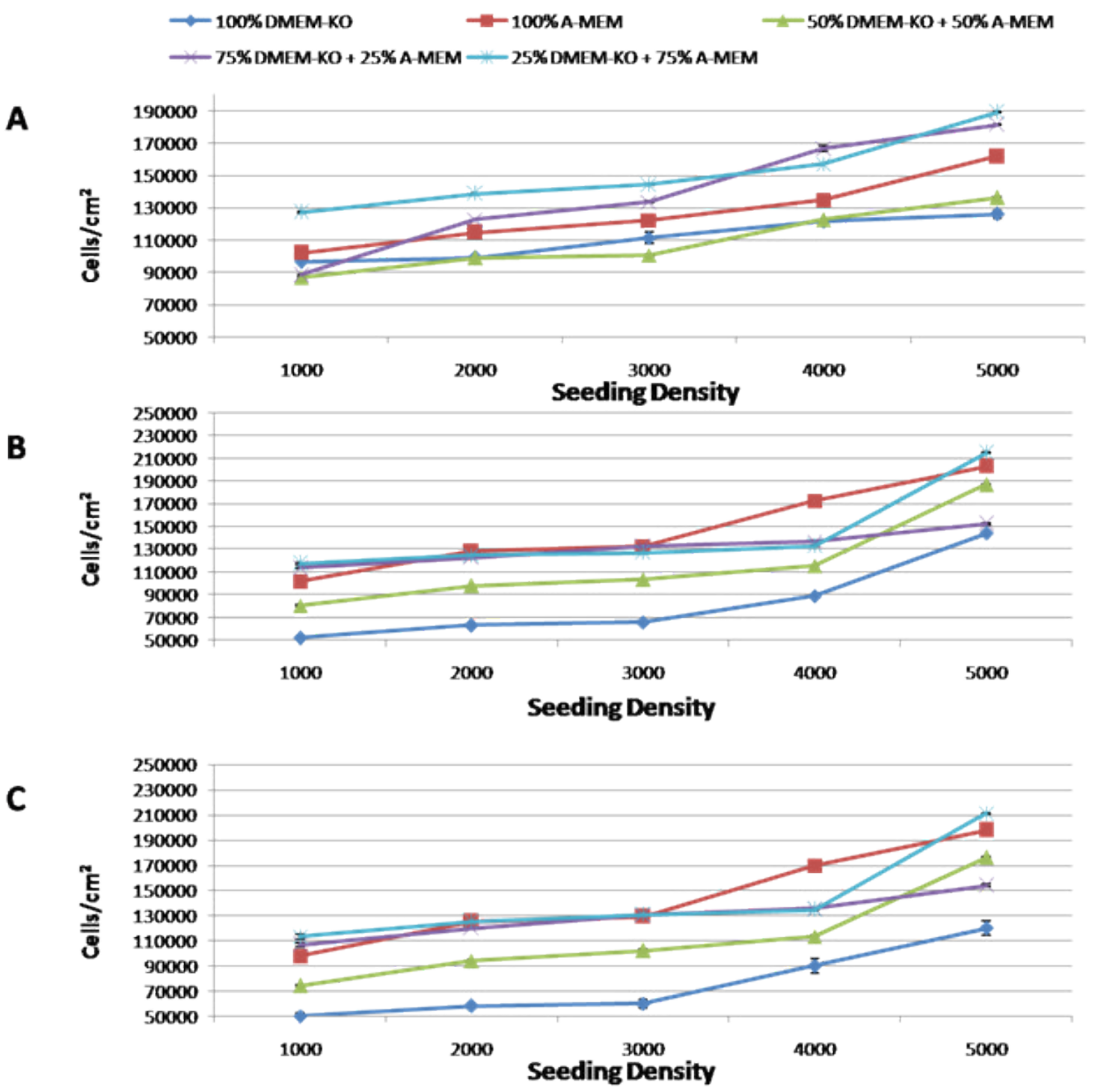

Figure 2: Growth curve of equine AD-MSCs cultured in different medium combinations and at different seeding densities at (A) passage 1, (B) passage 2 and (C) passage 3. Mean total cell yield was given as cell number per square centimetre. The results are average \pm S.E.M. of 3 culture replicates for each passage. 

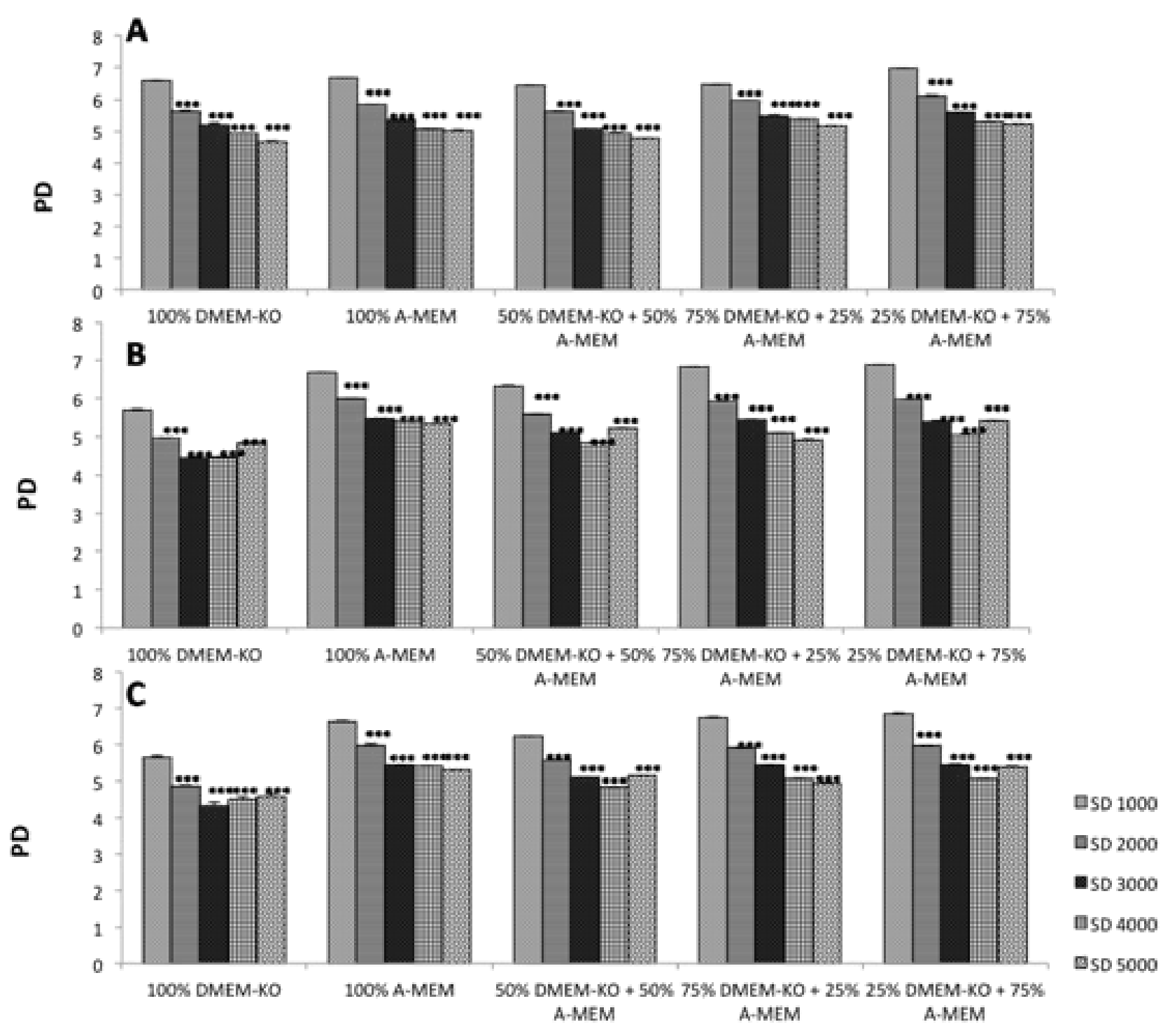

\section{Media Combinations}

Figure 3: Comparison of mean total population doublings for equine AD-MSCs cultured under different media conditions and at different seeding densities at (A) passage 1 , (B) passage 2 and (C) passage 3 . The results are average \pm S.E.M. of 3 culture replicates for each passage. ${ }^{* \star *}$ indicates $P<0.001$ compared to the 1000 seeding density.

which is significantly higher than the cells cultured in $\alpha$-MEM $(24.09 \mathrm{~h})$ or 25:75 DMEM-KO:a-MEM (26.84 h) (P<0.05) (Figure 4).

Overall results therefore showed that $\alpha$-MEM alone or in combination of 25:75 DMEM-KO: $\alpha$-MEM at the seeding density of $5000 \mathrm{cells} / \mathrm{cm}^{2}$ significantly improved proliferation rate with lesser cumulative population doublings that ensure significantly lower replicative senescence and in a shorter population doubling time. Therefore, in all subsequent experiments, 25:75 DMEM-KO:a-MEM medium combination was used for large scale expansion.

\section{In vitro multilineage differentiation potential of equine AD-} MSCs

There was no visual difference in multilineage differentiation capacity of equine AD-MSCs towards adipogenic, osteogenic and chondrogenic lineages in all different culture conditions tested in the experiments. Equine AD-MSCs retained their capacity to differentiate into adipocytes, osteoblasts and chondrocytes in all the different medium combinations and passages (Figure 1B-1D).

\section{Karyotype analysis}

To evaluate numerical and structural chromosomal abnormalities in equine $\mathrm{AD}$-MSCs during culture in various medium combinations and seeding densities, we performed G-band analysis on all samples (Figure 1E). None of the samples showed chromosomal abnormalities, indicating stable karyotypes in all the culture conditions and seeding densities.

\section{Immunophenotypic characterization of equine AD-MSCs}

All equine AD-MSCs cultured in different medium combinations 


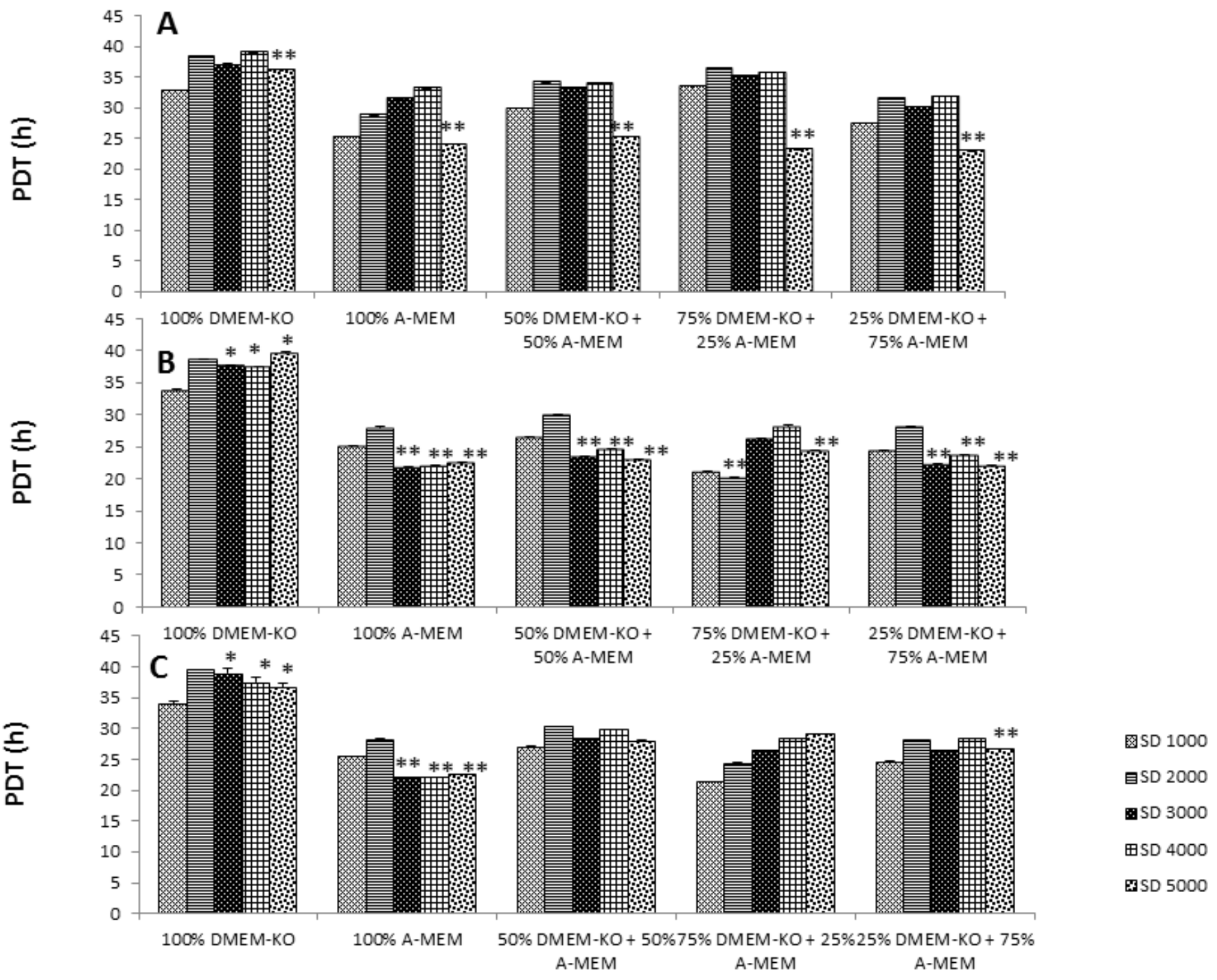

\section{Media Combinations}

Figure 4: Comparison of mean population doubling time (PDT) for equine AD-MSCs cultured in different medium combinations and seeding densities at (A) passage 1 , (B) passage 2 and $(C)$ passage 3 . The results are average \pm S.E.M. of 3 culture replicates for each passage. ${ }^{*}$ indicates $P<0.05,{ }^{*}$ indicates $P<0.01$ compared to the 1000 seeding density.

were negative for CD34, CD45 and HLA-DR and were consistently positive for the MSC markers CD44, CD90 and CD105, which are known to be expressed on MSCs (Figure 5). Percent expression of these markers was quite similar and also fairly stable for all the culture conditions in all the passages.

\section{Immunosuppression by equine AD-MSCs}

To assess the immunomodulatory properties of equine $\mathrm{AD}$ MSCs, we co-cultured MSCs with PBMCs in two different ratios (1:100 and 1:500) for 3 days, in the presence of PHA. We observed that the equine $\mathrm{AD}$-MSCs significantly inhibited $\mathrm{T}$-cell proliferation $(P<0.01)$ compared to the control. Further, T-cell proliferation dosedependently decreased as the number of MSCs added to each well decreased $(P<0.05)$ (Figure $1 \mathrm{~F})$.

Large scale expansion: The procedure for clinical grade large-scale expansion of equine AD-MSCs is illustrated in Figure 6. Qualified large-scale single-cell GMP-grade MCB (P-0) and WCB (P-1) of equine $\mathrm{AD}-\mathrm{MSCs}$ were generated as mentioned in Materials and Methods to provide virtually unlimited starting sources for final clinical grade cells for therapeutic use (P-2).

\section{Production of MCB}

Nucleated cells were seeded at a density of $85,000-100,000$ cells/ $\mathrm{cm}^{2}$ in a 1-cell stack $\left(636 \mathrm{~cm}^{2}\right)$ in optimized MSC culture medium 25:75 DMEM-KO: $\alpha$-MEM, since this was reported to be the optimum medium for proliferation and overall yield. An average of $107 \times 10^{6}$ \pm 10.25 equine $\mathrm{AD}$-MSCs were obtained from each 1 -chamber stack from each donor at passage- 0 (P-0) that gave on an average 107 vials of MCB containing 1 million cells each. Total 5 MCB from 5 independent healthy donors were prepared for this experiment. 

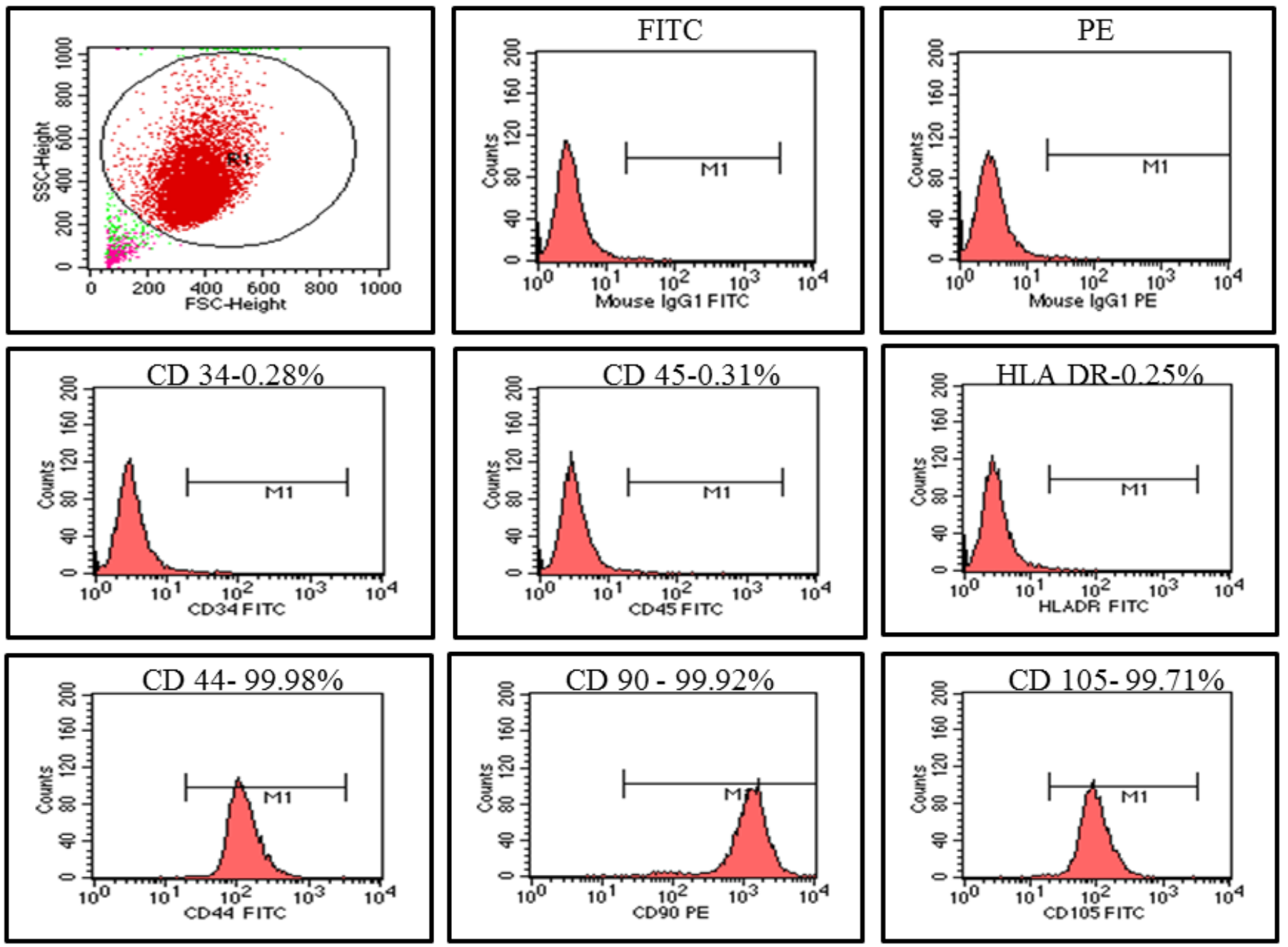

Figure 5: Representation of immunophenotypic analysis of equine AD-MSCs.

Preparation of working cell bank: Four vials of AD-MSCs from 4 different individual donors were thawed and mixed together and seeded in 5-chamber cell stack $\left(3180 \mathrm{~cm}^{2}\right)$ in an optimized MSC culture medium at the seeding density of 5000 cells $/ \mathrm{cm}^{2}$. An average of $6.5 \times$ $10^{8}$ cells of equine AD-MSC were obtained that gave on an average 65 frozen vials containing 10 million cells each.

\section{Preparation of production batch}

Four vials of WCB were thawed and seeded in 10 chamber stack in an optimized MSC cultured medium at the seeding density of 5000 cells/ $\mathrm{cm}^{2}$. On an average of $1.2 \times 10^{9}$ cells of equine AD-MSC were obtained. Thus, from each production batch 126 doses of 10 million each could be produced or 12 doses of 100 million each were produced. In each production batch multiple 10-chamber stacks could be included. The cells were frozen as off-the shelf product for clinical application. Upon thawing $95 \%$ post-thaw viability was observed.

Quality control: AD-MSCs at P-0 MCB were tested for viral screening and were found negative. AD-MSCs were tested for morphological phenotypes, cell surface markers, pluripotency, endotoxin, mycoplasma, karyotyping, sterility, trilineage differentiation and viability and found as per specification and met the release criteria.
Thawing of final product: Frozen vials of final product at $\mathrm{P}-2$ were removed and rapidly thawed at $37^{\circ} \mathrm{C}$ water bath for $1 \mathrm{~min}$. After dilution with Plasmalyte-A, cells were checked for viability using 7 -AAD marker. Viability of final thawed product was $90 \%$ and able to pass all the quality parameters without losing their stemness and differentiation capacity. Stability of the product showed that after thawing and dilution final product could be used within 3 hours.

\section{Pre-clinical safety study}

Fourteen days acute toxicity in Wistar rats and Swiss Albino mice by intravenous route at the dose of 20 million cells/ $\mathrm{kg}$ body weight was carried out and did not show any mortality and adverse reactions and was found to be safe.

\section{Discussion}

Regenerative medicine using stem cells is a new and rapidly growing area of research in the treatment of difficult-to-treat horse diseases. With this approach, stem cells are delivered into the damaged area where they stimulate regeneration and help repair damaged tissue and protect from reinjury [20]. For therapeutic intervention, a large number of clinical grade MSCs are required at an affordable cost. 


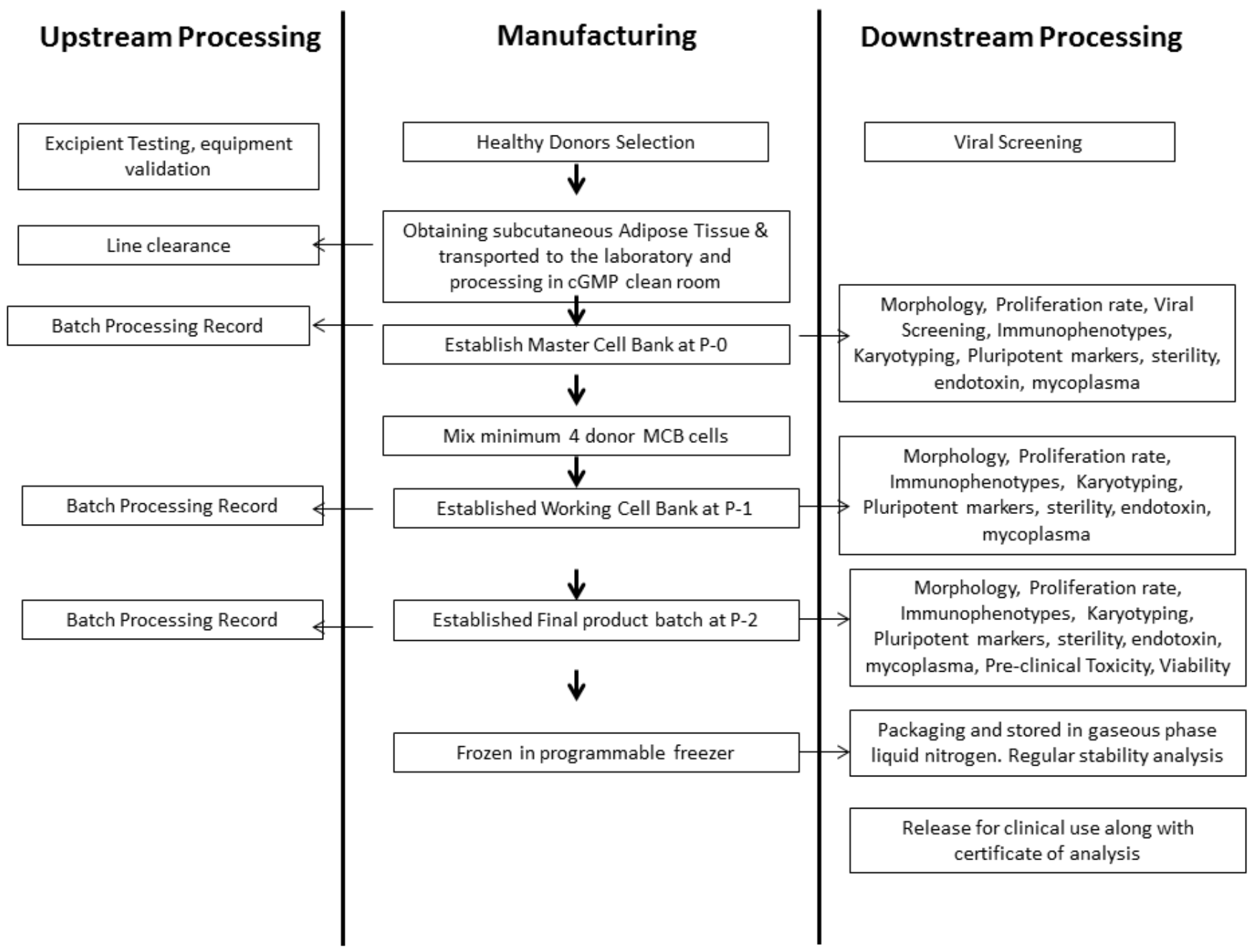

Figure 6: Typical plan for large-scale clinical grade ex vivo expansion of equine AD-MSCs.

Therefore, optimizing culture conditions by manipulating the culture niche is very important for large-scale expansion of MSC.

Here, we report a robust process of large-scale expansion of clinical grade minimally manipulated equine AD-MSCs. The qualified largescale single-cell GMP grade MCB and WCB of equine AD-MSCs were generated to provide virtually unlimited starting materials for expansion of clinical grade stem cells. We expanded equine ADMSCs from these banks upon thawing and generated more than 1.2 $\times 10^{9}$ cells in a 10 -chamber stack at passage 2 . The expansion process integrated a standardized healthy donor cell source, extensive quality control testing, pre-clinical safety analysis, efficacy parameters, viral screening and ability to be stored as an off-the-shelf product for immediate availability and use without further manipulation and without compromising the sterility of the cells. The final product at passage- 2 reported more than $90 \%$ post-thaw viability, a critical requirement for a product to be used in clinical trials or therapy (data not shown). Moreover, donor-derived AD-MSCs were negative for HLA-DR and also inhibited T-cell proliferation in PHA-stimulated PBMCs, suggesting its hypoimmunogenic and immunomodulatory properties and thus its safety for use in patients without tissue rejection was established.
Currently, most of the reports on equine stem cell therapy have demonstrated the use of autologous bone marrow mononuclear cells (BM-MNC) $[4,11,15]$ or adipose tissue-derived SVF [6,7]. Disadvantages of this procedure include the requirement of surgical intervention and post operative care of each horse and harvesting inconsistent cell numbers, which may result in inconsistent clinical outcomes. Therefore, large scale expansion of donor derived AD-MSC with minimally manipulated homogeneous population may be most effective for equine stem cell therapy and could be a good starting material for clinical application [21].

In this study, we compared different basal media to investigate which would best promote the expansion of the equine AD-MSCs without the cells losing their plasticity or stemness. Next, we studied the cells clinical grade expansion and their characteristic profiles to determine the safety of stem cells and their functional markers. The most commonly used media for culture of MSCs in equine are DMEMlow glucose (DMEM-LG), DMEM-high glucose (DMEM-HG), DMEM-F12 and TCM 199 [5,17-19,22-24].

In our previous studies, we had evaluated several basal medium such as DMEM-LG, DMEM-HG, DMEM-F12, DMEM-KO and $\alpha-M E M$ 
for isolation and expansion of MSCs derived from bone marrow, Wharton's Jelly, adipose tissue and dental pulp, and observed that DMEM-KO and $\alpha$-MEM were the most optimal culture conditions in terms of its proliferation, morphology, cell surface markers, population doublings, whereas cell cultured in DMEM-LG, DMEM-HG and DMEM-F12 showed lower cell proliferation, higher senescence and abnormal morphology [17-19]. Hence, in the current study we selected DMEM-KO and $\alpha$-MEM, alone or in combination in order to analyse synergistic effect at different seeding densities. We observed that the medium and seeding density influenced the proliferation rate, cell yield, population doubling and population doubling time. DMEM-KO is a reduced-osmolality basal medium, which was designed to improve embryonic stem (ES) cell morphology, reduce differentiation in culture and promote better human MSC growth [17-19]. We observed that the combination of 25:75 DMEM-KO/a-MEM was found to be the most ideal combination to achieve best parameters including the highest cell yield of 215,000 cells $/ \mathrm{cm}^{2}$ in a shorter PD and PDT and keeping all the safety parameters intact for clinical application, compared with the other medium combinations.

It is already documented that lower seeding densities resulted in faster cell proliferation compared with higher seeding densities. In one of the studies equine MSCs seeded at $100 \mathrm{cells} / \mathrm{cm}^{2}$ reached the target of 200 million cells, 4.1 days faster than the cells that were seeded at 5000 cells $/ \mathrm{cm}^{2}$ [25], whereas in an another study seeding density of $10^{5}$ cells/ $\mathrm{cm}^{2}$ was found optimum [5]. In this study, we observed that equine AD-MSCs seeded at 5000 cells $/ \mathrm{cm}^{2}$ in all medium combinations had a faster proliferation rate with shorter population doubling time and less cumulative population doublings.

Higher population doublings occurring at lower densities may affect the senescence of cells, cell characteristics and expression of transformation markers. However, previous findings showed that seeding densities did not affect cell characterization, as all cells had same cell marker characteristic profiles [26,27]. Determining the optimum seeding density for maximal expansion is useful for potential clinical applications because it will provide a cost-effective method that requires less time, thus decreasing the risk of contamination, loss of cell characteristics, cell transformation and chromosomal abnormalities.

The method used for large-scale manufacturing of clinical grade equine $\mathrm{AD}-\mathrm{MSC}$ are crucial. Under highly standardized culture conditions, continuous effect during long-term culture and replicative senescence has been reported $[28,29]$. Recently it was reported that long-term culture is associated with continuous changes in global gene expression profile starting from passage-3 onward $[30,16]$. Gene involved in these progressive changes were related to cell cycle, DNA replication, mitosis and DNA repair and were significantly down regulated. Long-term culture may also cause karyotyping abnormalities and up-regulation of transformation markers, rendering the cells useless for clinical applications. However, it is not yet clear how many passages or population doublings or senescence associated molecular changes are acceptable to grant optimal therapeutic effect. Considering this fact, MSCs should only be used for clinical purposes at an early passage of ex vivo culture; however, this may severely restrict the cell expansion process. In the present study, we have developed a process of large-scale expansion of clinical grade AD-MSCsat passage- 2 which are minimally manipulated and safe at pre-clinical toxicity studies and safe and efficacious at multi-centre clinical studies with 3 to 18 months follow-ups in tendon and ligament injuries, osteoarthritis and exercise induced pulmonary haemorrhage (data not shown).

In conclusion, this study shows that equine AD-MSCs may be expanded for clinicalscale production with minimal manipulation and without loss of functionality or stemness. This study further establishes the notion that manipulating the culture niche with the right growth medium and plating density could result in higher yields of cells without compromising the quality of the cells in terms of their content of early progenitors, growth rate, senescence and karyotype ensuring its complete safety for use in patient treatment (Figure 6).

\section{Conflict of interest}

The authors report no conflicts of interest and are responsible for the content and writing of the paper.

\section{Acknowledgements}

The authors thank Professor Dr HJ Thiel, Head of the Department of Microbiology at the Institute of Virology of the University of Giessen in Germany for viral screening of equine donors and the master cell bank.

\section{References}

1. Le BK (2003) Immunomodulatory effects of fetal and adult mesenchymal stem cells. Cytotherapy 5: 485-489.

2. Meirelles LS, Chagastelles PC, Nardi NB (2006) Mesenchymal stem cells resides in virtually all post-natal organs and tissues. J Cell Sci 119: 2204-2213.

3. Horwitz EM, Dominici M (2008) How do mesenchymal stromal cells exert their therapeutic benefit? Cytotherapy 10: 771-774

4. Baghaban Eslaminejad MR, Taghiyar L, Dehghan MM, Falahi F, Kazemi $\mathrm{MH}$ (2009) Equine marrow-derived mesenchymal stem cells: isolation, differentiation and culture optimization. J Vet Res 10: 1-11.

5. Coli A, Nocchi F, Lamanna R, lorio M, Lapi S, et al. (2011) Isolation and characterization of equine amnion mesenchymal stem cells. Cell Biol Int Rep 18: e00011.

6. Nixon AJ, Dahlgren LA, Haupt JL, Yeager AE, Ward DL (2008) Effect of adipose-derived nucleated cell fractions on tendon repair in horses with collagenase-induced tendinitis. Am J Vet Res 69: 928-937.

7. Braun J, Hack A, Weis-Klemm M, Conrad S, Treml S, et al. (2010) Evaluation of the osteogenic and chondrogenic differentiation capacities of equine adipose tissue-derived mesenchymal stem cells. Am J Vet Res71: 1228-1236.

8. Koch TG, Heerkens T, Thomsen PD, Betts DH (2007) Isolation of mesenchymal stem cells from equine umbilical cord blood. BMC Biotechnol 7: 26.

9. Smith RKW (2008) Stem cell therapy for tendon and ligament injuries in the horse: technique and outcome. Pferdeheillkunde 4: 513-518.

10. Carvalho AD, Badial PR, Alvarez LE, Yamada AL, Borges AS, et al. (2012) Equine tendonitis therapy using mesenchymal stem cells and platele concentrates: a randomized controlled trial. Stem Cell Res Ther 4: 85.

11. Godwin EE, Young NJ, Dudhia J, Beamish IC, Smith RK (2012) Implantation of bone marrow-derived mesenchymal stem cells demonstrates improved outcome in horses with overstrain injury of the superficial digital flexor tendon. Equine Vet J 44: 25-32.

12. Pittenger MF, Mackay AM, Beck SC, Jaiswal RK, Douglas R, et al. (1999) Multilineage potential of adult human mesenchymal stem cells. Science 284 143-147.

13. Wynn RF, Hart CA, Corradi-Perrini C, O'Neill L, Evans CA, et al. (2004) A small proportion of mesenchymal stem cells strongly expresses functionally active CXCR4 receptor capable of promoting migration to bone marrow. Blood 104 2643-2645.

14. Honczarenko M, Le Y, Swierkowski M, Ghiran I, Glodek AM, et al. (2006) Human bone marrow stromal cells express a distinct set of biologically functional chemokine receptors. Stem Cells 24: 1030-1041.

15. Hare JM, Fishman JE, Gerstenblith G, DiFede Velazquez DL, Zambrano JP et al. (2012) Comparison of allogeneic vs autologous bone marrow-derived mesenchymal stem cells delivered by transendocardial injection in patients with ischemic cardiomyopathy: the POSEIDON randomized trial. JAMA 308 2369-2379.

16. Wagner W, Bork S, Lepperdinger G, Joussen S, Ma N et al. (2010) How to track cellular aging of mesenchymal stromal cells? Aging 2: 224-230. 
Citation: Gowda S, Hari A, Chougule B, Reddy MK, Chandanan A, et al. (2013) Production of Good Manufacturing Practice Grade Equine Adiposederived Mesenchymal Stem Cells for Therapeutic Use. J Stem Cell Res Ther 3: 154. doi:10.4172/2157-7633.1000154

17. Pal R, Hanwate M, Jan M, Totey S (2009) Phenotypic and functional comparison of optimum culture condition for up scaling of bone marrow derived mesenchymal stem cells. J Tissue Eng Regen Med 3: 163-174.

18. Nekanti U, Rao VB, Bahirvani AG, Jan M, Totey S, et al. (2010) Long-term expansion and pluripotent marker array analysis of Wharton's jelly-derived mesenchymal stem cells. Stem Cells Dev 19: 117-130.

19. Govindasamy V, Ronald SV, Totey S, Din SB, Mustafa WM, et al. (2010) Micromanipulation of culture niche permits long-term expansion of dental pulp stem cells—an economic and commercial angle. In Vitro Cell Dev Biol 46: 764773

20. Estberg L, Stover SM, Gardner IA, Johnson BJ, Jack RA, et al. (1998) Relationship between race start characteristics and risk of catastrophic injury in thoroughbreds: 78 cases. J Am Vet Med Assoc 212: 544-549.

21. Adegani FJ, Langroudi L, Arefian E, Shafiee A, Dinarvand P, et al. (2013) Acomparison of pluripotency and differentiation status of four mesenchymal adult stem cells. Mol Biol Rep 40: 3693-3703.

22. Ranera B, Remacha AR, Álvarez-Arguedas S, Romero A, Vázquez FJ, et al. (2012) Effect of hypoxia on equine mesenchymal stem cells derived from bone marrow and adipose tissue. BMC Vet Res 8: 142.

23. Ranera B, Remacha AR, Álvarez-Arguedas S, Castiella T, Vázquez FJ, et al. (2013) Expansion under hypoxic conditions enhances the chondrogenic potential of equine bone marrow-derived mesenchymal stem cells. Vet $\mathrm{J} 195$ : 248-251.
24. Iacono E, Merlo B, Pirrone A, Antonelli C, Brunori L, et al. (2012) Effects of mesenchymal stem cells isolated from amniotic fluid and platelet-rich plasma gel on severe decubitus ulcers in a septic neonatal foal. Res Vet Sci 93: 14391440.

25. Vidal MA, Kilroy GE, Johnson JR, Lopez MJ, Moore MR, et al. (2006) Cell growth characteristics and differentiation frequency of adherent equine bone marrow-derived mesenchymal stromal cells: adipogenic and osteogenic capacity. Vet Surg 35: 601-610.

26. Bartmann C, Rohde E, Schallmoser K, Pürstner P, Lanzer G, et al. (2007) Two steps to functional mesenchymal stromal cells for clinical application. Transfusion 47: 1426-1435

27. Mochizuki T, Muneta T, Sakaguchi Y, Nimura A, Yokoyama A, et al. (2006) Higher chondrogenic potential of fibrous synovium- and adipose synoviumderived cells compared with subcutaneous fat-derived cells: distinguishing properties of mesenchymal stem cells in human. Arthritis Rheum 54: 843-853.

28. Wagner W, Ho AD, Zenke M (2010) Different Facets of Aging in Human Mesenchymal Stem Cells. Tissue Eng Part B Rev 2010.

29. Roobrouck VD, Ulloa-Montoya F, Verfaillie CM (2008) Self-renewal and differentiation capacity of young and aged stem cells. Exp Cell Res 314 : 1937-1944.

30. Wagner W, Horn P, Castoldi M, Diehlmann A, Bork S, et al. (2008) Replicative Senescence of Mesenchymal Stem Cells - a Continuous and Organized Process. PLoS One 5: e2213. 